\title{
Elektive Endoprothetik - Patientensteuerung nach Qualitätsergebnissen: Projekte der AOK Rheinland/Hamburg
}

\section{Mohrmann}

Qualitätsmanagement

Schlüsselwörter

Elektive Hüft-TEP und Knie-TEP

Patientensteuerung

Bewertung der Ergebnisqualität

QSR als Beurteilungskriterium

Patientenbefragung

Keywords

Drimary total prosthetic hip and knee replacements

patient management appraisal of outcome quality QSR as appraisal criterion

patient survey
Institut

AOK Rheinland/Hamburg,

Düsseldorf

Bibliografie

DOI $10.1055 / \mathrm{s}-0029-1242674$

Dtsch Med Wochenschr 2009;

134: S318 - (c) Georg Thieme

Verlag KG Stuttgart · New York .

ISSN 0012-0472

Korrespondenz

Matthias Mohrmann,

Dipl.-Kfm.

AOK Rheinland/Hamburg

Die Gesundheitskasse

Kasernenstraße 61

40213 Düsseldorf

Tel. 0211/8791-2570

eMail matthias.mohrmann@

rh.aok.de
Der Versorgungssektor Krankenhaus verfügt in Deutschland über erheblich höhere Kapazitäten als in vergleichbaren Ländern der OECD. Die Reduzierung der durch die staatliche Krankenhausplanung vorgegebenen Restriktionen hinsichtlich des Versorgungsauftrags der Krankenhäuser öffnet Leistungsbereiche für Anbieter, die in diesen Feldern bisher nicht tätig waren. Verstärkt wird diese Entwicklung durch die Änderungen im Vertragsarztrecht im Jahr 2009, in deren Folge niedergelassene Vertragsärzte ohne besondere Anforderungen gleichzeitig auch als angestellter Arzt im Krankenhaus tätig werden können.

Ein Beispiel für die Wirkungen dieser erweiterten Handlungsoptionen für Krankenhäuser ist die Endoprothetik. War beispielsweise im Bundesland Nordrhein-Westfalen die Durchführung elektiver endoprothetischer Eingriffe in der Vergangenheit auf eine überschaubare Zahl besonders ausgewiesener Orthopädien beschränkt, drängen jetzt viele Krankenhäuser mit chirurgischen Fachabteilungen und ohne besondere orthopädische Expertise auf den Markt. Dies auch deshalb, weil die Belegung der Krankenhäuser in vielen der ambulanten Leistungserbringung nahen Bereichen rückläufig ist und Kompensationsmöglichkeiten in anderen Leistungssegmenten gesucht werden. Niedergelassene Orthopäden sehen in diesem Feld für sich zusätzliche Betätigungschancen und werden als Operateur im Krankenhaus bei von ihnen selbst eingewiesenen Patienten aktiv.

Aus Sicht der AOK Rheinland/Hamburg ist ein freies Spiel der Kräfte im Sektor der stationären Krankenhausversorgung problematisch, vor allem deshalb, weil trotz aller Bemühungen in der Vergangenheit nur unzureichende Transparenz hinsichtlich der Qualität der Leistungserbringung besteht. Dies gilt natürlich in erster Linie für den Patienten, der sich im Regelfall an der Empfehlung seines niedergelassenen Arztes orientiert, im Zweifel also des Arztes, der ihn operieren wird. Die Frage der Orientierung stellt sich aber in gleicher Weise dem niedergelassenen Arzt, der aus rein medizinischer Sicht zu einzelnen Krankenhäusern seine Empfehlungsentscheidung abgeben will.

Die der gesetzlichen Krankenversicherung vorliegenden Daten erlauben - zumindest innerhalb bestimmter Grenzen - Rückschlüsse auf die Qualität der Leistungserbringung. Das „QSR“Projekt des WIdO stellt die Basis unserer Ansätze dar. Um die Frage zu beantworten, ob qualitativ hochwertige Leistungen erbracht werden, können darüber hinaus weitere Informationsquellen genutzt werden. Hierzu gehören unter anderem Informationen der BQS (Bundesgeschäftsstelle Qualitätssicherung), Qualitätsberichte der Krankenhäuser sowie Befragungen von Patienten und weiterversorgenden Leistungserbringern.

Erste Ansätze zur Nutzung dieser unterschiedlichen Datenquellen sowie sich daraus ergebende Perspektiven u.a. im Hinblick auf selektiv-vertragliche Elemente werden am Beispiel der elektiven Endoprothetik dargestellt.

Autorenerklärung: Der Autor erklärt, dass keine relevanten finanziellen Verbindungen in Bezug auf dieses Manuskript bestehen. 